\title{
A novel approach to a rare case of non-islet cell hypoglycaemia
}

\section{R K Dharmaputra1', K L Wan², N Samad1', M Herath1,3, J Wong1,4, S Sarlos ${ }^{1,4,5}$, S R Holdsworth ${ }^{6}$ and $\mathbf{N}$ Naderpoor ${ }^{1,3}$}

1Diabetes and Vascular Medicine Department, 2Monash Health Pathology, Monash Health, Victoria, Australia, ${ }^{3}$ Monash Centre for Health Research and Implementation, School of Public Health and Preventive Medicine, Monash University, Victoria, Australia, ${ }^{4}$ Department of Endocrinology, Monash Health, Victoria, Australia, 5 Department of Medicine, School of Clinical Sciences, Monash University, Victoria, Australia, and ${ }^{6}$ Department of Immunology, Monash Health, Victoria, Australia
Correspondence should be addressed to $\mathrm{N}$ Naderpoor Email negar.naderpoor@monash. edu

\section{Summary}

Insulin autoimmune syndrome (IAS) is a rare cause of non-islet cell hypoglycaemia. Treatment of this condition is complex and typically involves long-term use of glucocorticoids. Immunotherapy may provide an alternative in the management of this autoimmune condition through the suppression of antibodies production by B-lymphocyte depletion. We present a case of a 62-year-old male, with refractory hypoglycaemia initially presenting with hypoglycaemic seizure during an admission for acute psychosis. Biochemical testing revealed hypoglycaemia with an inappropriately elevated insulin and C-peptide level and no evidence of exogenous use of insulin or sulphonylurea. Polyethylene glycol precipitation demonstrated persistently elevated free insulin levels. This was accompanied by markedly elevated anti-insulin antibody (IA) titres. Imaging included CT with contrast, MRI, pancreatic endoscopic ultrasound and Ga 68-DOTATATE position emission tomography (DOTATATE PET) scan did not reveal islet cell aetiology for hyperinsulinaemia. Maintenance of euglycaemia was dependent on oral steroids and dextrose infusion. Complete resolution of hypoglycaemia and dependence on glucose and steroids was only achieved following treatment with plasma exchange and rituximab.

\section{Learning points:}

- Insulin autoimmune syndrome (IAS) should be considered in patients with recurrent hyperinsulinaemic hypoglycaemia in whom exogenous insulin administration and islet cell pathologies have been excluded.

- Biochemical techniques play an essential role in establishing high insulin concentration, insulin antibody titres, and eliminating biochemical interference. High insulin antibody concentration can lead to inappropriately elevated serum insulin levels leading to hypoglycaemia.

- Plasma exchange and B-lymphocyte depletion with rituximab and immunosuppression with high dose glucocorticoids are effective in reducing serum insulin levels and hypoglycaemia in insulin autoimmune syndrome (IAS).

- Based on our observation, the reduction in serum insulin level may be a better indicator of treatment efficacy compared to anti-insulin antibody (IA) titre as it demonstrated greater correlation to the frequency of hypoglycaemia and to hypoglycaemia resolution. 


\section{Background}

Hypoglycaemia is infrequently encountered in nondiabetic individuals. At the time of presentation, reversible and secondary causes of hypoglycaemia such as starvation, medication misadministration, hepatic decompensation, and use of insulin or oral hypoglycaemic agents should be excluded. Once reversible causes have been ruled out, endogenous causes of hyperinsulinaemia are sought.

Anti-insulin antibody (IA)-mediated hypoglycaemia or insulin autoimmune syndrome (IAS) is a rare cause of non-islet cell hypoglycaemia (1). It is postulated that IAs bind to endogenous insulin, and enhance the plasma halflife, leading to hyperinsulinaemia. Insulin would later dissociate from the autoantibody in a disorganised pattern and provoke hypoglycaemia $(2,3)$. In contrast, anti-insulin receptor antibody acts synergistically with insulin and causes hypoglycaemia through direct activation of the insulin receptor (2). As the clinical presentation of either mechanism is hypoglycaemia, biochemical techniques are necessary to ascertain the underlying pathophysiology. Consequently, adjunctive approaches have been used to measure IA concentration. Most commonly used techniques include immunoprecipitation with polyethylene glycol (PEG) of macro-analytes, heterophilic antibody interference, dilution studies, confirmation by an alternative platform and the gold standard gel filtration chromatography (GFC) (4). GFC can demonstrate the presence of macroinsulin, which correlates well with IA by illustrating an insulin peak in the immunoglobulin mass area.

Previously, the treatment of IAS has been limited to the use of long-term glucocorticoids. Advances in immunology have facilitated exploration of other treatment options, including plasmapheresis, which has been effectively used in a number of cases (5).

\section{Case presentation}

A 62-year-old male mechanic was brought to the emergency department by his brother, who was concerned about behavioural change over a few months. He was reported to have fixed ideation regarding the loss of penile function and had been self-medicating with an alternate daily dose of sildenafil $100 \mathrm{mg}$ and tadalafil $20 \mathrm{mg}$. There was no prior psychiatric history and his past medical history includes erectile dysfunction, gastro-oesophageal reflux, and chronic obstructive airway disease. He occasionally felt hot and cold at night and reported a 'funny feeling' during sleep. Collateral history from his brother revealed frequent episodes of hand tremors that were often associated with hunger. There were no clinical features suggestive of adrenal insufficiency.

Clinical examination on arrival was unremarkable and there were no signs of hypoglycaemia. His BMI was $23.5 \mathrm{~kg} / \mathrm{m}^{2}$. The patient was admitted to the Psychiatric Unit for further management of a possible delusional disorder and was commenced on regular antipsychotics, paliperidone and valproate. On day 14 of admission, he had a generalised tonic-clonic seizure. Point of care blood glucose reading was $0.7 \mathrm{mmol} / \mathrm{L}$, which was confirmed on a venous sample.

\section{Investigations}

The hormone profile recorded demonstrated an inappropriately elevated level of C-peptide and insulin. Counter-regulatory hormones including, cortisol and growth hormone, had risen appropriately (Table 1). Sulphonylurea screening was negative.

Endoscopic ultrasound of the pancreas revealed no lesions suggestive of insulinoma or nesidioblastosis. CT scan of the chest, abdomen, and pelvis as well as a dedicated MRI of the pancreas were unremarkable. Ga-68 DOTATATE

Table 1 Insulin, C-peptide, and Pituitary hormones levels.

\begin{tabular}{|c|c|c|c|c|c|}
\hline & Day $1(7: 55 h)$ & Day $1(23: 00 \mathrm{~h})$ & Day $1(23: 45$ h) & Day 2 (6:00 h) & Reference interval \\
\hline Glucose (mmol/L) & & 1.8 & 1.2 & 7.5 & $3.0-6.0$ \\
\hline Insulin (mU/L) & & 272.9 & 250.9 & 167.5 & $1.9-23.0$ \\
\hline C-peptide (nmol/L) & 2.59 & 2.58 & 2.48 & 2.64 & $0.26-1.39$ \\
\hline Cortisol (nmol/L) & 257 & & 438 & & 185-625 (a.m.) \\
\hline FSH (IU/L) & & & & 4.0 & $2.2-16.0$ \\
\hline LH (IU/L) & & & & 1.6 & $2.0-11.0$ \\
\hline Growth hormone (mU/L) & & 2.2 & 36.9 & & $0-3.0$ \\
\hline IGF-1 (nmol/L) & & & & 26.2 & $9.8-27.8$ \\
\hline Testosterone (LCMS) (nmol/L) & & & & 18 & $10.0-25.0$ \\
\hline
\end{tabular}


PET scan did not detect any focal lesions suggestive of insulinoma.

Flow cytometry and bone marrow aspiration and biopsy results were normal. CSF analysis was performed to investigate autoimmune encephalitis as the cause of his altered mental state. Anti-glutamic acid decarboxylase (GAD), N-methyl-D-aspartate (NMDA) receptor, and voltage-gated K channel-complex (VGKC) antibodies were not detected.

Serum insulin was measured using an automated 'sandwich' chemiluminescent immunoassay (Beckman Coulter UniCel D×I 800). The Access Ultrasensitive Insulin assay did not interfere with C-peptide (up to a concentration of $20000 \mathrm{pmol} / \mathrm{L}$ ) and to an extremely minimal extent, with proinsulin (minute reduction, that is, $-0.26 \%$ when $4000 \mathrm{pmol} / \mathrm{L}$ of proinsulin is added to a calibrator which has a value of $70 \mathrm{pmol} / \mathrm{L}$ ). Serum IA titre was measured by immunoprecipitation of I125-(A14)-monoiodinated insulin and antihuman IgG with protein-A and protein-G Sepharose. The amount of radioactivity was proportional to the concentration of IA.

Exogenous insulin administration was excluded due to markedly elevated levels of both insulin and C-peptide levels. Insulin assay interference was investigated with serial dilution, use of alternative platforms (Siemens and Roche), immunoprecipitation with PEG and heterophile antibodies. While the free insulin concentration decreased following PEG treatment (Table 2), it remained abnormally elevated above the reference range.

We hypothesised, that the patient's recurrent hypoglycaemia was secondary to IAS due to the persistently elevated serum IA levels while biochemical and radiologic investigations did not reveal evidence of islet cell pathology.

\section{Treatment}

Severe hypoglycaemia with seizure was treated according to protocol with $50 \mathrm{~mL}$ of intravenous $50 \%$ dextrose and intramuscular $1 \mathrm{mg}$ glucagon. However, hypoglycaemia persisted despite further treatment with $60 \mathrm{mg}$ oral glucose, resulting in commencement of an infusion of $5 \%$ dextrose (Fig. 1). Five hours following the seizure, he was asymptomatic, but point of care capillary glucose was only $2.5 \mathrm{mmol} / \mathrm{L}$; this was confirmed with venous blood glucose level (Table 1 and Fig. 1).

For 2 weeks following the first seizure the patient remained glucose-infusion dependent despite concurrent daily oral intake of up to 12 Mega joules including 225-240 g carbohydrate and $100 \mathrm{mg}$ protein daily. Prednisolone $10 \mathrm{mg} \mathrm{BD}$ was commenced to reduce overnight intravenous glucose requirements.

Following the demonstration of elevated IA titres, four sessions of plasma exchange (PLEX) and two infusions of rituximab were administered, in consultation with Immunology department. Biochemical analysis before and after each PLEX showed a reduction in serum insulin levels following each session (Fig. 2 and Table 2). Furthermore, CD19 and CD20 count were undetectable following rituximab infusion.

Prednisolone dose was temporarily increased to $50 \mathrm{mg}$ daily to facilitate rapid de-escalation of dextrose infusion. Intravenous dextrose was ceased following the second rituximab infusion, as no further hypoglycaemic events were recorded (Fig. 3). Prednisolone was also rapidly down titrated to $20 \mathrm{mg} / \mathrm{d}$ within 10 days after the first rituximab infusion. Hypoglycaemia was deemed resolved by the absence of hypoglycaemic events for a week after complete cessation of intravenous glucose. The patient's care was transitioned to the Psychiatry Unit with a plan for ongoing blood glucose monitoring and weekly steroid tapering.

Table 2 Clinical and biochemical response to PLEX and rituximab.

\begin{tabular}{|c|c|c|c|c|c|c|c|}
\hline Date & PLEX & Rituximab & $\begin{array}{l}25 \% \text { Dextrose } \\
\text { infusion }\end{array}$ & Prednisolone & Insulin (mU/L) & Insulin post-PEG (mU/L) & $\begin{array}{c}\text { Anti-insulin } \\
\text { antibody }(\mathrm{U} / \mathrm{mL})\end{array}$ \\
\hline 13/09/19 (8:17 h) & & & & & 2828.8 & 86.8 & $>17$ \\
\hline 20/09/19 (13:40 h) & $1 \mathrm{st}$ & & $40 \mathrm{~mL} / \mathrm{h}$ & $10 \mathrm{mg}$ BD & 297.2 & 90.4 & \\
\hline 24/09/19 (10:00 h) & 2nd & & $40 \mathrm{~mL} / \mathrm{h}$ & $10 \mathrm{mg} B D$ & 2322.4 & 138.3 & \\
\hline 24/09/19 (13:45 h) & & & $40 \mathrm{~mL} / \mathrm{h}$ & & 256.7 & 95.8 & \\
\hline 01/10/19 & & & $25 \mathrm{~mL} / \mathrm{h}$ & & & & \\
\hline $02 / 10 / 19$ & $3 r d$ & & $25 \mathrm{~mL} / \mathrm{h}$ & & & & \\
\hline $04 / 10 / 19$ & & $700 \mathrm{mg}$ & $25 \mathrm{~mL} / \mathrm{h}$ & 50 mg Daily & & & $>17$ \\
\hline 07/10/19 & & & $0-20 \mathrm{~mL} / \mathrm{h}$ & 35 mg Daily & & & \\
\hline 08/10/19 & & & Ceased & & & & \\
\hline 11/10/19 (7:00 h) & 4 th & & & 25 mg Nocte & 89.3 & 25.3 & $>17$ \\
\hline $14 / 10 / 19$ & & $700 \mathrm{mg}$ & & 20 mg Nocte & & & \\
\hline
\end{tabular}




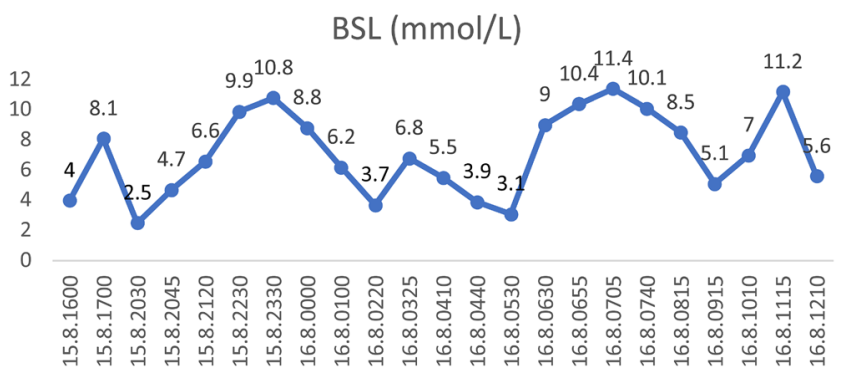

Figure 1

Glycaemic trend following onset of seizure demonstrating hypoglycaemia despite dextrose infusion 15 August 2019 to 16 August 2019.

\section{Outcome and follow-up}

Serum IA level remained elevated despite significant reduction in serum insulin and the resolution of hypoglycaemia. The patient's capillary blood glucose was monitored four times daily for a month and there were no further episodes of hypoglycaemia. Following discharge, he was seen at the Endocrinology Outpatient Clinic. Despite ongoing delusions, he denied any further consumption of PDE Inhibitors. Over 3 months after immunotherapy, prednisolone was gradually ceased with no further hypoglycaemia or seizures. Despite the resolution of hypoglycaemia, he has recently been diagnosed with early onset dementia.

\section{Discussion}

Due to its rarity, there are currently no guidelines or consensus on how to best manage patients with IAS. At the time of writing this case report, there were no publications comparing the effectiveness of various treatment modalities for IAS.

In this case, the diagnosis of IAS was made after multiple investigations including Ga-68 DOTATATE PET scan and endoscopic ultrasound of the pancreas failed to demonstrate

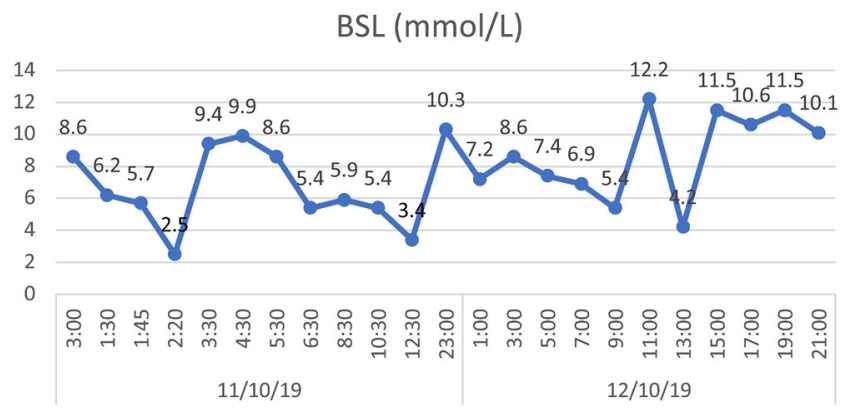

Figure 2

Glycaemic trend following 4th session of PLEX on 11 October 2019 (dextrose infusion ceased, prednisolone $25 \mathrm{mg}$ nocte).

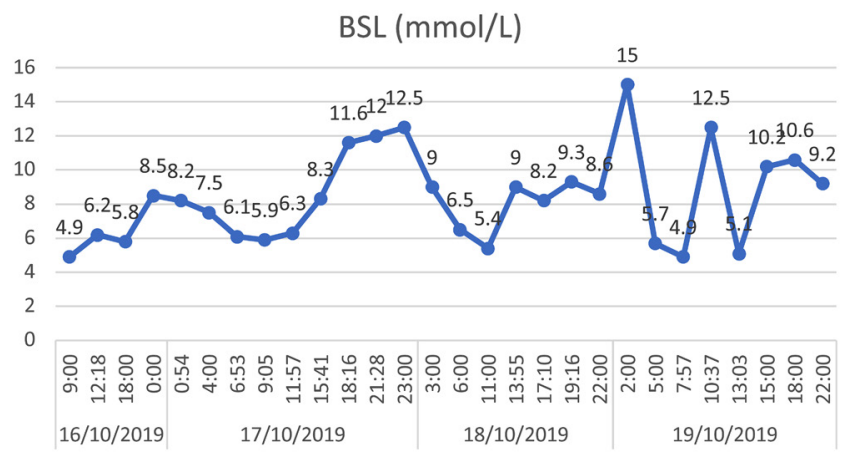

Figure 3

Glycaemic trend following 4th cycle of PLEX and 2nd rituximab infusion (dextrose infusion ceased, prednisolone $20 \mathrm{mg}$ nocte).

evidence of islet cell aetiology for hypoglycaemia. This diagnosis was further supported by the elevated IA titres associated with hyperinsulinaemic hypoglycaemia and significant clinical response to immunotherapy. We found no evidence of haematological malignancy or other autoimmune conditions. In the absence of underlying malignancy, the cause of autoantibody production may be either medication-related or idiopathic in nature. Sildenafil, a sulphur-containing medication, is a possible causative agent; however, there is currently no commercial laboratories offering serum sildenafil level analysis in Australia.

Hypoglycaemia has previously been described in association with the consumption of non-prescription illegal erectile dysfunction pills containing a combination of sildenafil and glibenclamidein China (6). Hypoglycaemia in these settings were thought to occur due to unintentional ingestion of sulfonylurea rather than an autoimmune reaction. Our patient had no history of overseas travel and obtained his medications within Australia. Furthermore, sulphonylurea screen, using high-performance liquid chromatography with UV/VIS detection, was negative. The assay detects glipizide, gliclazide, glibenclamide, chlorpropamide, tolbutamide, and tolazamide. Sulphurcontaining medications, including sildenafil, have historically been implicated in autoantibody production (1). It is argued that the sulphur molecule may enhance the immunogenicity of insulin by reducing sulphydryl bonds connecting insulin chains (7). However, no previous case of IAS has been reported in relation to sildenafil usage.

Hypoglycaemia in the context of IAS occurs where there is an imbalance between insulin supply and demand. IA fragments bind to insulin resulting in prolonged plasma half-life which is evidenced by the reversed insulin to C-peptide ratio of $>1(2,3,8)$. The unpredictable dissociation of insulin molecules irrespective of blood glucose concentrations will then lead to recurrent hypoglycaemic 
events. Recurrent mild hypoglycaemic attacks maybe missed, both because of non-specific symptoms such as tremor, hunger and diaphoresis and also due to reduced hypoglycaemia awareness over time. This may explain why hypoglycaemia was not recognised earlier in our case.

Conversely, persistent hyperglycaemia due to type B insulin resistance syndrome (IRS) has also been described in individuals with IA . Type B IRS typically results from the blocking of insulin receptor by anti-insulin receptor antibody. However, individuals with IA may also develop insulin resistance where IA binds to insulin molecules preventing receptor binding. Immunotherapy has been demonstrated to be effective in managing both IAS and type B IRS (9).

Advances in immunotherapy have aided the management of autoantibody related conditions, including IAS. Chen et al. reported a case of IA mediated hypoglycaemia secondary to a low-grade B-Cell nonHodgkin lymphoma (5). This was managed with plasma exchange, rituximab, and glucocorticoids which resulted in reduced frequency of hypoglycaemia and a $30 \%$ reduction in IA titre 6 weeks following therapy. However, the patient continued to experience mild hypoglycaemia despite low GI diet and acarbose in the 2-year follow up period.

In our patient, treatment of IAS with rituximab, an anti-CD20 MAB- induced disease remission. However, even though our patient rapidly had all circulating CD20 B-cells depleted, there was no correlation between the reduction of IA titres and disease remission. The persistently elevated IA level may be attributed to the immunoglobulin variability and assay interference as the antibody may be either IgG with a variable ratio of kappa to lambda light chains or IgA antibody; furthermore, the antibodies can also be monoclonal or polyclonal (2).

In addition, the efficacy of rituximab in treating IAS could be underpinned by mechanisms other than downregulation of humoral immunity by CD20 depletion. The rituximab in ANCA-associated vasculitis (RAVE) study compared rituximab infusion in patients with aggressive ANCA vasculitis with standard therapy and demonstrated 'not inferiority'. ANCA vasculitis is driven by humoral immunity, but no correlation was found between ANCA titres and disease remission. However, a rise in $\mathrm{T}$ regulatory cell count was shown in patients treated with rituximab that was associated with disease remission (10). The rise in $\mathrm{T}$ regulatory cells in response to rituximab has also been reported in animal models of rheumatoid arthritis and autoimmune diabetes. $\mathrm{T}$ regulatory cell upregulation may therefore have played a role in our patient's clinical response to rituximab.
In conclusion, immunotherapy with plasma exchange in conjunction with rituximab infusion can be an effective treatment for insulin autoimmune syndrome (IAS). Furthermore, serum insulin level, in this case, was a better prognostic marker than IA titre. This is because antibody binding affinity pattern, and therefore, the effect of antibody titre on insulin concentration can be unpredictable due to variable immunoglobulins. Although not measured in this study, the number of rituximabinduced $\mathrm{T}$ regulatory cells may also be a better marker for remission of IAS than IA titre.

\section{Patient's perspective}

Psychiatric involvement in this case provided a unique and detailed insight into this patient's perspective in relation to his diagnosis. The patient understood that he was admitted to the hospital due to 'excessive use of erectile dysfunction pills'. He often felt frustrated by the frequency of blood glucose monitoring performed and associated sleep interruption, although this was ameliorated with the introduction of continuous glucose monitoring. He stated that he felt depressed because 'nothing works out for him;' however, he appreciated the need to remain in hospital due to frequent hypoglycaemia. During his hospital stay, he developed significant fear of hypoglycaemia and became agitated when his meals did not arrive on time.

\section{Declaration of interest}

The authors declare that there is no conflict of interest that could be perceived as prejudicing the impartiality of this case report.

\section{Funding}

This work did not receive any specific grant from any funding agency in the public, commercial, or not-for-profit sector.

\section{Patient consent}

Publication consent was obtained from patient's daughter who is also his carer in the community.

\section{Author contribution statement}

The authors thank and acknowledge the contribution of members of The Monash Health Endocrinology, Immunology, Haematology, and Medical Imaging Department who were involved in the care of this patient. The authors thank Prof Peter Fuller, from Endocrinology Department and A/ Prof Zhong Lu and A/Prof James Doery from Monash Pathology for their guidance and valuable comments.

\section{Acknowledgements}

The authors also acknowledge Melbourne Health Pathology (The Royal Melbourne Hospital), Australian Clinical Labs and Melbourne Pathology for insulin and anti-insulin antibodies measurements.

\section{References}

1 Hirata Y, Ishizu H, Ouchi N, Motomura M, Abe M \& Hara Y. Insulin autoimmunity in a case with spontaneous hypoglycemia. Journal of the Japan Diabetes Society 197013 312-320. (https://doi.org/10.11213/ tonyobyo1958.13.312) 
Endocrinology

Diabetes \& Metabolism

CASE REPORTS
R K Dharmaputra and

others
A novel approach to IAS

management
ID: 21-0040; July 2021

DOI: 10.1530/EDM-21-0040
2 Lupsa BC, Chong AY, Cochran EK, Soos MA, Semple RK \& Gorden P. Autoimmune forms of hypoglycemia. Medicine 200988 141-153. (https://doi.org/10.1097/MD.0b013e3181a5b42e)

3 Ismail AA. The insulin autoimmune syndrome (IAS) as a cause of hypoglycaemia: an update on the pathophysiology, biochemical investigations and diagnosis. Clinical Chemistry and Laboratory Medicine 201654 1715-1724. (https://doi.org/10.1515/cclm-2015-1255)

4 Church D, Cardoso L, Bradbury S, Clarke C, Stears A, Dover A, Halsall D \& Semple R. Diagnosis of insulin autoimmune syndrome using polyethylene glycol precipitation and gel filtration chromatography with ex vivo insulin exchange. Clinical Endocrinology 201786 347-353. (https://doi.org/10.1111/cen.13179)

5 Chen AX, Beligaswatte A, White G \& Burt MG. Rituximab for treatment of refractory insulin autoimmune syndrome associated with non-Hodgkin B-cell lymphoma. Clinical Endocrinology 201991 230-232. (https://doi.org/10.1111/cen.13998)

6 Poon WT, Lam YH, Lee HHC, Ching CK, Chan WT, Chan SS, Lai CK, Tse ML, Chan AYW \& Mak TWL. Outbreak of hypoglycaemia: sexua enhancement products containing oral hypoglycaemic agent. Hong Kong Medical Journal 200915 196-200.
7 Cryer PE, Axelrod L, Grossman AB, Heller SR, Montori VM, Seaquist ER, Service FJ \& Endocrine Society. Evaluation and management of adult hypoglycemic disorders: an Endocrine Society clinical practice guideline. Journal of Clinical Endocrinology and Metabolism 200994 709-728. (https://doi.org/10.1210/jc.20081410)

8 Cappellani D, Macchia E, Falorni A \& Marchetti P. Insulin autoimmune syndrome (Hirata disease): a comprehensive review fifty years after its first description. Diabetes Metabolic Syndrome and Obesity 20201 963-978. (https://doi.org/10.2147/DMSO.S219438)

9 Greenfield JR, Tuthill A, Soos MA, Semple RK, Halsall DJ, Chaudhry A \& O'Rahilly S. Severe insulin resistance due to anti-insulin antibodies: response to plasma exchange and immunosuppressive therapy. Diabetic Medicine 200926 79-82. (https://doi.org/10.1111/j.14645491.2008.02621.x)

10 Stone JH, Merkel PA, Speira R, Seo P, Langford CA, Hoffman GS, Kallenberg CGM, St Claire EW, Turkiewicz A, Tchao NK, et al. Rituximab versus cyclophosphamide for ANCA-associated vasculitis. New England Journal of Medicine 2010363 221-232. (https://doi. org/10.1056/NEJMoa0909905)

Received in final form 12 May 202

Accepted 14 June 2021 